\title{
Erratum to: Optimal differentiation of high- and low-grade glioma and metastasis: a meta-analysis of perfusion, diffusion, and spectroscopy metrics
}

\author{
Jurgita Usinskiene $^{1}$ • Agne Ulyte ${ }^{2}$ - Atle Bjørnerud ${ }^{3,4}$ • Jonas Venius ${ }^{1}$. \\ Vasileios K. Katsaros $^{5}$ - Ryte Rynkeviciene ${ }^{1} \cdot$ Simona Letautiene $^{1,2} \cdot$ Darius Norkus $^{1}$. \\ Kestutis Suziedelis $^{1} \cdot$ Saulius Rocka ${ }^{2,6}$ - Andrius Usinskas ${ }^{7}$ - Eduardas Aleknavicius ${ }^{1,2}$
}

Published online: 21 May 2016

(C) Springer-Verlag Berlin Heidelberg 2016

Erratum to: Neuroradiology (2016) 58:339-350

DOI 10.1007/s00234-016-1642-9

We note that the following Acknowledgment was inadvertently left out of "Optimal differentiation of high and low grade glioma and metastasis: a meta-analysis of perfusion, diffusion and spectroscopy metrics"

Acknowledgment

This research was funded by a grant (No. MIP-015/2014) from the Research Council of Lithuania.

The online version of the original article can be found at http://dx.doi.org/ $10.1007 / \mathrm{s} 00234-016-1642-9$.

Jurgita Usinskiene

jurgita.usinskiene@gmail.com

1 National Cancer Institute, Radiology Center, Santariskiu 1, LT-08660 Vilnius, Lithuania

2 Faculty of Medicine, Vilnius University, Vilnius, Lithuania

3 Department of Physics, Oslo University Hospital, Oslo, Norway

4 The Intervention Centre, Oslo University Hospital, Oslo, Norway

5 General Anti-Cancer and Oncological Hospital "St. Savvas", Athens, Greece

6 Neuroangiosurgery Center, Faculty of Medicine Vilnius University, Vilnius, Lithuania

7 Department of Electronic Systems, Vilnius Gedimino Technical University, Vilnius, Lithuania 